\title{
Proteinosis alveolar, un hallazgo incidental en un estudio preoperatorio con respuesta insuficiente al lavado pulmonar completo
}

\author{
F. J. GONZÁLEZ-BARCALA, S. BLANCO-GONZÁLEZ1, L. VALDÉS-CUADRADO, \\ J. M. GARCÍA-PRIM ${ }^{1}$, A. L. GOLPE-GÓMEZ, R. LEDO-ANDIÓN ${ }^{1}$
}

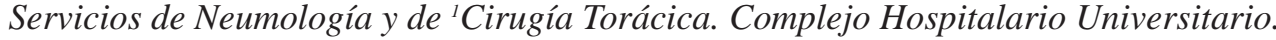
Santiago de Compostela.

ALVEOLAR LIPOPROTEINOSIS, AN INCIDENTAL FINDING DURING AN UNRELATED PRE-SURGICAL STUDY WITH INADEQUATE RESPONSE TO COMPLETE PULMONARY WASHING

\section{RESUMEN}

La lipoproteinosis alveolar (LPA) es una enfermedad pulmonar difusa poco frecuente, acumulándose un exceso de fosfolípidos en la vía aérea distal, cuyo síntoma más frecuente es la disnea.

Paciente de 35 años de edad en la que se observan, como hallazgo incidental durante un estudio preoperatorio, infiltrados pulmonares en una radiografía de tórax. Con las exploraciones físicas y complementarias realizadas en el Servicio de Neumología, no se obtiene un diagnóstico definitivo, por lo que la paciente es remitida al Servicio de Cirugía Torácica para realizar videotoracoscopia diagnóstica. El estudio de las biopsias obtenidas demuestra la presencia de LPA.

La LPA es una enfermedad poco frecuente que suele plantear dificultades diagnósticas, y en múltiples ocasiones requiere biopsia pulmonar para obtener el diagnóstico definitivo. El pronóstico global de la enfermedad es excelente con tratamiento. La terapia más segura y efectiva es el lavado pulmonar completo (LPC), que en nuestra paciente fue necesario a los 7 meses del diagnóstico por presentar deterioro clínico así como en los estudios de función pulmonar realizados, con pobre respuesta al mismo. En los 6 meses siguientes se realiza nuevo LPC, sin resultados satisfactorios; iniciándose entonces tratamiento con factor estimulante de colonias de granulocito-macrófagos (GM-CSF), con respuesta favorable.

PALABRAS CLAVE: Lipoproteinosis alveolar. Biopsia pulmonar. Lavado pulmonar completo. Factor estimulante de colonias de granulocito-macrófagos.
ABSTRACT

The alveolar lipoproteinosis $(A L P)$ is a rare pulmonary disease, characterized by an excess of phospholipids in the distal airway, and the most symptom of which is dyspnea.

35 years old patient in whom we observed incidentally pulmonary infiltrations in a chest X-ray during a unrelated pre-surgical study. We could not make a definitive diagnosis after further investigations carried out in the Division of Respiratory Medicine. We, therefore, sent this patient to the Division of Chest Surgery for a complementary video-thoracoscopy. Biopsy showed presence of ALP.

The ALP is a rare disease that originates diagnosis difficulties, and that often needs lung biopsies to confirm its diagnosis. Once treated, its prognosis is excellent. The safest and most effective treatment is a complete pulmonary washing, that, in our patient, was required 7 months after diagnosis as this patient presented clinical deterioration and worsening in the lung function studies. This treatment did not achieve the expected goal. In the subsequent 6 months, we repeated the same treatment and failed again. We then started a treatment with granulocytemacrophage-colony stimulating factor (GM-CSF) and obtain good response.

KEY WORDS: Alveolar lipoproteinosis. Lung biopsy. Complete pulmonary washing. Granulocyte-macrophage-colony stimulating factor.

González-Barcala FJ, Blanco-González S, Valdés-Cuadrado L, García-Prim JM, Golpe-Gómez AL, Ledo-Andión R. Proteinosis Alveolar, un hallazgo incidental en un estudio preoperatorio con respuesta insuficiente al lavado pulmonar completo. An Med Interna (Madrid) 2003; 20: 410412.

\section{INTRODUCCIÓN}

La lipoproteinosis alveolar (LPA) es una enfermedad pulmonar difusa poco frecuente, con una incidencia estimada entre uno y dos casos por millón de habitantes (1), descrita inicialmente por Rosen y cols en 1958 (2)

Se produce por un exceso de fosfolípidos con positividad para tinción con ácido periódico de Schiff en la vía aérea distal, sin reacción inflamatoria ni alteración de la arquitectura pulmo- nar $(3,4)$. En la patogénesis de esta enfermedad se invocan procesos de disminución de la eliminación de fosfolípidos por los macrófagos alveolares (3), o exceso de producción de los mismos por los neumocitos tipo II, o ambos procesos simultáneamente $(1,4,5)$. Kitamura considera la posibilidad de encontrarnos ante una enfermedad autoinmune (6). En una publicación reciente se observa, entre los pacientes con esta enfermedad, elevada prevalencia de fumadores y de exposición laboral a polvos inhalados (7).

Trabajo aceptado: 14 de enero de 2003 
Se distinguen principalmente dos formas de LPA: una primaria, y otra forma secundaria asociada a la exposición a sustancias químicas, polvos minerales, drogas como el busulfan o el clorambucil, neoplasias hematológicas u otras situaciones de inmunodeficiencia. También está descrita una forma hereditaria autosómica recesiva $(1,3,4)$.

La disnea es el síntoma más frecuente, habitualmente de esfuerzos moderados, pudiendo también presentarse con tos, fiebre o febrícula, o no presentar ningún síntoma hasta que se desarrolla alguna infección respiratoria sobreañadida $(1,8)$.

\section{CASO APORTADO}

Paciente de 35 años de edad entre cuyos antecedentes personales destacan dos cesáreas, ser fumadora de cigarrillos de 20 paquetes/año y haber presentado una pitiriasis rosada de Gibert por lo cual su dermatólogo le ha indicado hidratación cutánea tópica. Trabajó previamente en una factoría de conservas de pescado durante varios años, estando en el momento del diagnóstico sin ocupación laboral. No refiere alergias conocidas.

En un estudio preoperatorio para ligadura de trompas, se observan infiltrados pulmonares en la radiografía de tórax, por lo cual es remitida al Servicio de Neumología. Interrogada intencionadamente refiere tos con expectoración escasa desde hace menos de un año y disnea de esfuerzos moderados, grado 1 de la MRC (9), que atribuía a su hábito tabáquico. La paciente no refiere contacto con polvos orgánicos ni inorgánicos, ni episodios de incremento de disnea ni sintomatología en relación con ninguna exposición.

En la exploración física mostraba constantes vitales dentro de la normalidad sin que se apreciaran hallazgos relevantes en la exploración por aparatos.

En las exploraciones complementarias se aprecia un hemograma dentro de la normalidad, bioquímica normal salvo colesterol 249 mg/dL (150-220), amilasa 79 uI/L(20-70), LDH 337 UI/L (115-326); inmunoglobulinas: IgM: $221 \mathrm{mg} / \mathrm{dL}$ (50-200), IgE: $143 \mathrm{uI} / \mathrm{mL}$ (10130); proteinograma normal; anticuerpos antinucleares negativos. VSG: $1^{\mathrm{a}}$ hora $10 \mathrm{~mm}, 2^{\mathrm{a}}$ hora: $20 \mathrm{~mm}$. Gasometría arterial basal: pO2: 93 mmHg, pCO2: 42 mmHg, pH: 7.43, CO3H: 27. Exploración funcional respiratoria: FVC: $2620 \mathrm{ml}(75 \%)$, FEV1: $2300 \mathrm{ml}(83 \%)$, FEV1/FVC: $88 \%$ con test de broncodilatadores negativo, TLC: 2810 ml (56\%), RV: 660 (44\%), RV/TLC: 24 (78\%). La difusión mostró una DLCO de $21.40 \mathrm{ml} / \mathrm{min} / \mathrm{mmHg}(71 \%)$ con D/VA de 5.04 (118\%). ECG: ritmo sinusal a 70 1/min. Serología de mycoplasma, chlamydia, legionella y coxiella burnetti: negativo. BAAR en esputo: negativo. Fibrobroncoscopia: árbol bronquial con mucosa normal sin otros hallazgos relevantes. Se realiza aspirado general, biopsia bronquial y transbronquial. La citología de aspirado general mostró macrófagos alveolares de citoplasma espumoso y células cilíndricas ciliadas de epitelio respiratorio, negativa para malignidad. Los cultivos para bacterias, micobacterias y hongos fueron negativos. La biopsia bronquial no demuestra alteraciones histopatológicas y la transbronquial fue informada como parénquima pulmonar sin alteraciones. El ecocardiograma mostró cámaras de dimensiones normales, función ventricular conservada, sin datos de hipertensión pulmonar. Radiografía de tórax: múltiples lesiones parenquimatosas de aspecto algodonoso de predominio parahiliar. TAC de tórax de alta resolución (TAC-AR): extensas áreas pulmonares bilaterales parcheadas de opacidad en "ground glass", de predominio en regiones superiores y centrales, con engrosamiento uniforme de los septos interlobulares de las áreas afectas, constituyendo estas dos alteraciones combinadas el patrón de "crazy paving". No se aprecian adenopatías hiliares ni mediastínicas. Ecografía abdominal: sin alteraciones.

No pudiendo obtener un diagnóstico definitivo se remite a la paciente al Servicio de Cirugía Torácica. Se realiza videotoracoscopia tomándose biopsias de lóbulo superior derecho (LSD) y lóbulo inferior derecho (LID) que son informadas por el Servicio de Anatomía Patológica como proteinosis alveolar.

Inicialmente se mantiene a la paciente en observación, pero ante el deterioro clínico y analítico se realiza lavado pulmonar completo (LPC) en dos ocasiones, con pobre respuesta; por lo cual se inicia tratamiento con GM-CSF, manteniéndose la paciente, durante los 4 meses transcurridos desde entonces, estable, aunque ante la persistencia de insuficiencia respiratoria requiere oxigenoterapia domiciliaria.

\section{DISCUSIÓN}

La LPA plantea frecuentemente dificultades diagnósticas $(1,4,6,8,10)$. Para ello, además de la historia clínica, exploración física y datos analíticos, la TAC-AR y el estudio del material obtenido en el lavado broncoalveolar (BAL) que habitualmente presenta un aspecto lechoso, constituido fundamentalmente por fosfolípidos, sobre todo lecitina, además de otras proteínas $(1,4,8,12,14)$. La biopsia transbronquial puede ser diagnóstica en ocasiones, aunque dada la afectación parcheada característica de esta enfermedad una biopsia que muestra parénquima pulmonar normal no es excluyente. Kitamura propone un método diagnóstico basado en autoanticuerpos contra el factor estimulante de colonias de macrófagosgranulocitos (6).

La biopsia pulmonar sigue siendo el "gold standard" en el diagnóstico de las enfermedades pulmonares intersticiales, ya sea por vídeotoracoscopia o por biopsia pulmonar abierta (4), aunque se describen en la literatura casos en que la biopsia pulmonar no fue diagnóstica, pero sí lo fue el BAL (11).

En el diagnóstico diferencial inicial se incluyen enfermedades inflamatorias con afectación alveolar. En el caso que nos ocupa, dado el curso clínico y los resultados obtenidos en las exploraciones físicas y complementarias realizadas se han planteado como primeras posibilidades la BONO, neumonía eosinófila, neumonía por hipersensibilidad o proteinosis alveolar. Asimismo se valora la posibilidad de neumonitis intersticial idiopática o sarcoidosis en fases iniciales, la hemorragia alveolar y la linfangioleiomiomatosis; siendo la histiocitosis $\mathrm{X}$ y la amiloidosis menos probables por su afectación fundamentalmente intersticial.

En la LPA la exploración física puede resultar anodina aunque lo más frecuente son los crepitantes inspiratorios, observándose acropaquias en casi la mitad de los pacientes $(1,8,12)$.

Es casi constante la elevación de la LDH. Se ha sugerido que la combinación de LDH elevada y aumento de la diferencia alvéoloarterial de oxígeno orienta principalmente a dos diagnósticos: LPA o neumonía por Pneumocystis carinii (4, 12). En la exploración funcional respiratoria predomina un patrón restrictivo, con reducción de volúmenes y de la capacidad de difusión $(1,4)$.

En el presente caso la exploración funcional respiratoria evidencia alteración restrictiva moderada, apreciándose una discreta elevación de la LDH sin aumento en la diferencia alveolo-arterial de oxígeno. Los estudios endoscópicos tampoco fueron concluyentes. Atribuimos la escasa alteración en todos estos datos a que la paciente fue remitida a nuestro servicio por un hallazgo casual en el contexto de un estudio preoperatorio y por lo tanto correspondía a una fase inicial de la enfermedad.

En el estudio radiológico simple del tórax la forma de presentación más frecuente es con infiltrados parcheados bilate- 
rales, de predominio en bases; aunque en un 20\% de los casos se limitan a un único lóbulo. En la TAC-AR de tórax presenta el patrón denominado "crazy paving”, que inicialmente fue considerado específico de la LPA, aunque ahora conocemos que también aparece en otras enfermedades como el carcinoma bronquioloalveolar, la neumonía lipoidea, neumonía por pneumocystis carinii o infección por citomegalovirus; sin que se describan patrones patognomónicos de LPA en la TAC-AR $(1,4,13)$.

El pronóstico global de la proteinosis alveolar es excelente con tratamiento, aunque difícil de predecir $(1,7)$. En la serie de Goldstein, el $46 \%$ de los pacientes no requerían lavado pulmonar completo (LPC) en el tiempo de seguimiento (8). Por otra parte, se han publicado casos de remisión espontánea sin tratamiento $(10,15,16)$. De ser necesario algún tratamiento el LPC es el más seguro y efectivo (1), siendo la administración de GM-CSF una alternativa en casos seleccionados $(17,18)$. Las infecciones oportunistas son la complicación más frecuente, y deben tratarse de forma específica. En el caso presente se decidió tratamiento conservador inicialmente dado que la paciente se encontraba asintomática. Siete meses después del diagnóstico ha presentado incremento significativo de la disnea, deterioro en los estudios de gasometría arterial y exploración funcional respiratoria, por lo cual ha sido remitida a otro centro hospitalario para la realización de LPC, mejorando tras este tratamiento tanto clínicamente como en los parámetros de los estudios complementarios; pasando la presión parcial de oxígeno en sangre arterial de $60 \mathrm{mmHg}$ antes de realizar el LPC a $84 \mathrm{mmHg}$ después del mismo, la capacidad vital forzada de $1.980 \mathrm{ml}$ a $2.260 \mathrm{ml}$, el volumen espiratorio forzado en el primer segundo de 1.800 a $1.970 \mathrm{ml}$, la capacidad de transferencia de monóxido de carbono (DLCO) desde el $44 \%$ del predicho al $63 \%$, y la DLCO corregida para volumen alveolar efectivo del $74 \%$ al $125 \%$ del predicho. Tres meses después del LPC la paciente vuelve a presentar deterioro clínico y analítico, sin poder demostrar infección respiratoria, por lo cual se realiza nuevo LPC, mejorando transitoriamente; pero reingresando de nuevo en insuficiencia respiratoria al mes de realizado el lavado. Ante la pobre respuesta a los dos LPC, se inicia tratamiento con GM-CSF, manteniéndose desde entonces estable, aunque requiere oxígeno domiciliario ante la persistencia de insuficiencia respiratoria.

\section{Bibliografía}

1. Shah PL, Hansell D, Lawson PR, et al. Pulmonary alveolar proteinosis: clinical aspects and current concepts on pathogenesis. Thorax 2000; 55: 67-77.

2. Rosen SH, Castleman B, Liebow AA. Pulmonary alveolar proteinosis. N Engl J Med 1958; 258: 1123-42.

3. Griese M. Pulmonary surfactant in health and human lung diseases: state of the art. Eur Respir J 1999; 13: 1455-76.

4. Wang BM, Stern EJ, Schmidt RA, et al. Diagnosing pulmonary alveolar proteinosis. A review and an update. Chest 1997; 111: 460-6.

5. Prakash UBS, Barham SS, Carpenter HA, et al. Pulmonary alveolar phospholipoproteinosis: experience with 34 cases and a review. Mayo Clin Proc 1987; 62: 499-518.

6. Kitamura T, Uchida K, Tanaka N, et al. Serological diagnosis of idiopatic pulmonary alveolar proteinosis. Am J Respir Crit Care Med 2000; 162: $658-62$

7. Briens E, Delaval P, Mairesse MP, et al. lipoprotéinose alvéolaire pulmonaire. Rev Mal Respir 2002; 19: 166-82.

8. Goldstein LS, Kavuru MS, Curtis-McCarthy P, et al. Pulmonary alveolar proteinosis. Clinical features and outcomes. Chest 1998; 114: 1357-62.

9. Mahler DA, Wells CK. Evaluation of clinical methods for rating dyspnea. Chest 1998; 93: 580-6.

10. García Río F, Alvarez-Sala R, Caballero P, et al. Six cases of pulmonary alveolar proteinosis: presentation of unusual associations. Monaldi Arch
Chest Dis $1995 ; 50: 1,12-5$.

11. Crocker HL, Pfitzner J, Doyle YR, Hague WM et al. Pulmonary alveolar proteinosis: two contrasting cases. Eur Respir J 2000; 15: 426-9.

12. Agustí AGN, Solé M. Mujer de 37 años con síndrome de Cushing y disnea progresiva de un año de evolución. Med Clín (Barc) 1997; 108: 109-16.

13. Lee KN, Levin DL, Webb R et al. Pulmonary alveolar proteinosis High-Resolution CT, Chest Radiographic, and Functional Correlations Chest 1997; 111: 989-95.

14. Kedia RK, Sullivan A, Stephens M, et al. A breathless female. Eur Respir J 1999; 13: 207-9.

15. Haddad PG, Pankey GA. Pulmonary alveolar proteinosis: a case with spontaneous resolution. J La State Med Soc 1969; 121: 365-76.

16. Canto MJ, Vives MA, Carmona F, et al. Successful pregnancy after spontaneous remission of familial pulmonary alveolar proteinosis. Eur $\mathbf{J}$ Obstet Gynecol Reprod Biol 1995; 63: 191-3

17. Seymour JF, Presneill JJ, Schoch OD, et al. Therapeutic efficacy of granulocyte-macrophage Colony-Stimulating Factor in patients with idiopathic adquired alveolar proteinosis. Am J Respir Crit Care Med 2001; 163: 524-31.

18. Kavuru MS, Sullivan EJ, Piccin R, et al. Exogenous granulocitemacrophage colony-stimulating factor administration for pulmonary alveolar proteinosis. Am J Respir Crit Care Med 2000; 161: 1143-48. 